\title{
Contaminação e detecção de material orgânico por método da eletrorresistividade
}

Carlos H. Alexandrino; Israel César Santana Júnior; Jefferson Batemarque Gomes; Paulo Henrique Gomes Silva; Rafael Miquelão Gottardi. Universidade Federal dos Vales do Jequitinhonha e Mucuri - Campos Avançado do Mucuri.

\begin{abstract}
Copyright 2014, SBGf - Sociedade Brasileira de Geofísica. Este texto foi preparado para a apresentação no VI Simpósio Brasileiro de Geofísica, Porto Alegre, 14 a 16 de outubro de 2014. Seu conteúdo foi revisado pelo Comitê Técnico do VI SimBGt, mas não necessariamente representa a opinião da SBGf ou de seus associados. É proibida a reprodução total ou parcial deste material para propósitos comerciais sem prévia autorização da SBGf.
\end{abstract}

\section{Resumo}

Apresenta-se nesse trabalho a descrição de uma experiência para simular o efeito da presença da solução salina no subsolo. O método utilizado foi 0 da eletrorresistividade, com a emprego da técnica do Caminhamento Elétrico. Os resultados obtidos mostram que a resistividade aparente é fortemente influenciada pela presença de materiais tóxicos, como por exemplo, o chorume.

\section{Introdução}

Um estudo sobre contaminação de solo tem o intuito de prever anomalias que possam afetar o meio ambiente, com isso foram utilizados métodos da eletrorresistividade para mapear e consequentemente amenizar os danos causados.

Com o aumento do consumo humano, tem se tornado frequente, o acúmulo de lixo em aterros sanitários, por exemplo, em que o chorume produzido pode escoar e alcançar um lençol freático ou um aquífero. Outro exemplo seria o chorume liberado pela decomposição de corpos em um cemitério (que geralmente se situa em locais elevados, como morros), tendo também o impacto sobre aquífero e/ou lençóis freáticos (GALLAS et al 2005).

Dentre os fatores que potencializam a utilização dos métodos da eletrorresistividade, para fins de estudo litológicos, um dos mais importantes é a diferença de potencial presente no solo. Essa condição é que permite a imensa possibilidade de aplicação do método em estudos ambientais e hidrogeológicos, pois normalmente as substâncias contaminantes geram líquidos com alta concentração em sais (GALLAS et al. 2005).

\section{Área de Estudo}

Esse estudo foi realizado em uma área dentro do Campus da UFVJM - Universidade Federal dos Vales do Jequitinhonha e Mucuri, no Município de Teófilo Otoni/MG, através de uma simulação do evento investigado, fazendo a abertura de uma vala para simular um aqüífero fraturado, conforme descrito na figura (1).

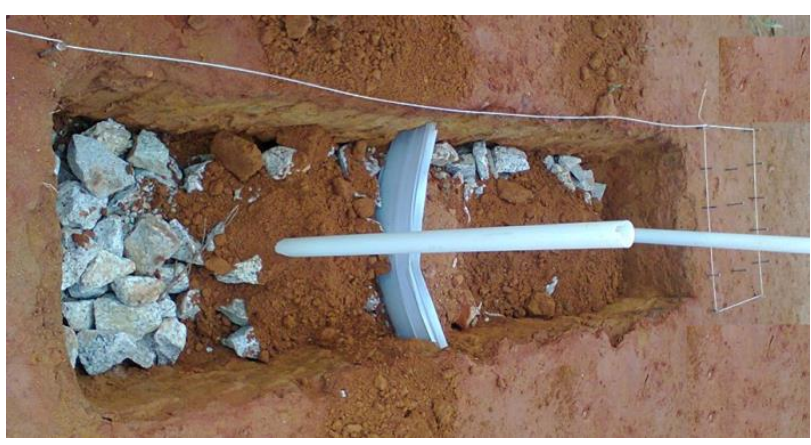

Figura 1 - foto da preparação da experiência

Em termos geomorfológicos, o município está compreendido na unidade "Planaltos Dissecados do Leste de Minas", em uma "zona de pontões", caracterizada por formas de relevo evoluídas por processos de erosão diferencial e descamação concêntrica. (MAGALHÃES JR., A.; MOREIRA, P. F.).

\section{Fundamentação Teórica}

Pertencente ao grupo dos métodos geoelétricos, a eletrorresistividade, é um método geofísico cujo princípio está baseado na determinação da resistividade elétrica dos materiais que, juntamente com a constante dielétrica e a permeabilidade magnética, expressam fundamentalmente as propriedades eletromagnéticas dos solos e rochas. Os diferentes tipos de materiais existentes no ambiente geológico, apresentam como uma de suas propriedades fundamentais o parâmetro físico resistividade elétrica, o qual reflete algumas de suas características servindo para caracterizar seus estados, em termos de alteração, faturamento, saturação, etc., e até identificá-los litologicamente, sem necessidade de escavações físicas" (BRAGA, A. C. O., 2007).

Existem duas técnicas principais do método da eletrorresistividade. São elas, a Sondagem Elétrica Vertical - SEV e o Caminhamento Elétrico - CE. A técnica da SEV consiste em uma sucessão de medidas de um parâmetro geoelétrico, efetuadas, a partir da superfície do terreno, mantendo-se uma separação crescente entre os eletrodos de emissão de corrente e recepção de potencial. Quando os eletrodos são alinhados na superfície do terreno de maneira simétrica, e durante a sucessão de medidas, a direção do arranjo e o centro do dipolo de recepção de potencial permanecem fixos. "Existem dois tipos principais de arranjos para o desenvolvimento da técnica da SEV: Shlumberger e Wenner." (BRAGA, A. C. O., 2007).

Neste estudo, optou-se pela técnica do CE. Essa técnica está relacionada à resistividade, que é obtida a partir de medidas feitas na superfície do local analisado, sendo 
investigada sua variação na horizontal confirmando sua profundidade. Sendo assim, o CE é usado mais comumente na aquisição de dados para confecção e plotagem de mapeamentos de subsolo e subsuperfícies, uma vez que a resistividade de rochas e materiais litológicos, assim como água, e resíduos poluentes podem ser facilmente identificados (MOREIRA et al., 2009).

Utilizou-se nesse trabalho o arranjo dipolo-dipolo em que a profundidade de investigação depende diretamente do espaçamento entre os eletrodos, sendo Z a profundidade do nível investigado e $R$ o espaçamento entre os centros dos eletrodos, temos que $Z=R / 2$, onde $R$ é a distância entre os centros dos dipolos considerado ( $A B$ e $M N$ ). Entretanto, na prática, essa relação é vista com maior coerência se for utilizado um valor de $R$ aproximadamente igual a um quarto da profundidade do nível investigado.

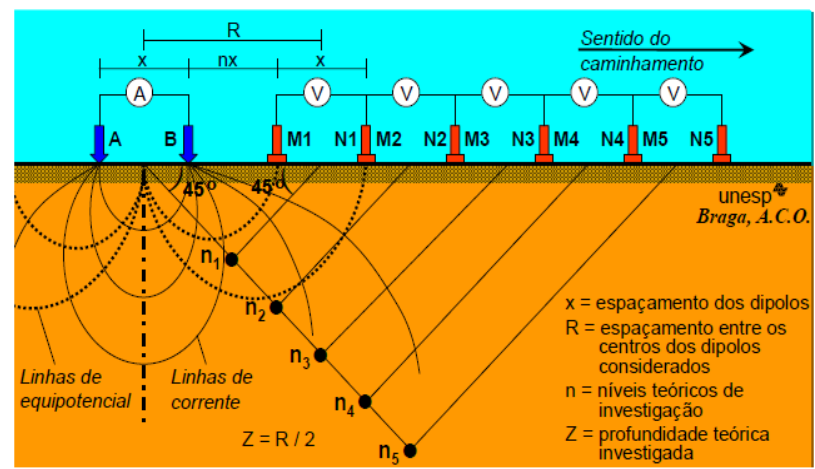

Figura 2 - Arranjo de desenvolvimento Dipolo-Dipolo CE. Fonte: (BRAGA, A. C. O., 2007).

A expressão utilizada para calcular a resistividade aparente é:

$$
\rho_{a}=k \frac{\Delta V}{I}
$$

Obtida por meio da emissão de uma corrente $l$, emitida através de dois eletrodos $A$ e $B$ e a diferença de potencial $\Delta \mathrm{V}$, usualmente medida através dos eletrodos de potencial ditos $\mathrm{M}$ e $\mathrm{N}$. Com isso é possível obter a resistividade aparente $\rho_{a}$.

$$
k=\frac{2 \pi}{\frac{1}{\overline{A M}}-\frac{1}{\overline{B M}}-\frac{1}{\overline{A N}}+\frac{1}{\overline{B N}}}
$$

onde $K$ é o fator geométrico que depende das distâncias entre os eletrodos de coerente $(A$ e $B$ ) e de potencial $(M$ e N), I é a corrente gerada entre os eletrodos de corrente e $\Delta \mathrm{V}$ a diferença de potencial estabelecida entre $\mathrm{M}$ e $\mathrm{N}$.

\section{Materiais e Métodos}

Para a execução do estudo, utilizou-se dois tubos de PCV, preenchidos de espuma, com orifícios ao redor de suas bases, de forma que permitissem, gradualmente, a vazão da solução ionizada. Após a abertura da vala, foi usado um pedaço de forro PCV (figura 3) para a divisão da vala em duas células, uma para recebimento da solução ironizada e outra a água comum, para comparação.

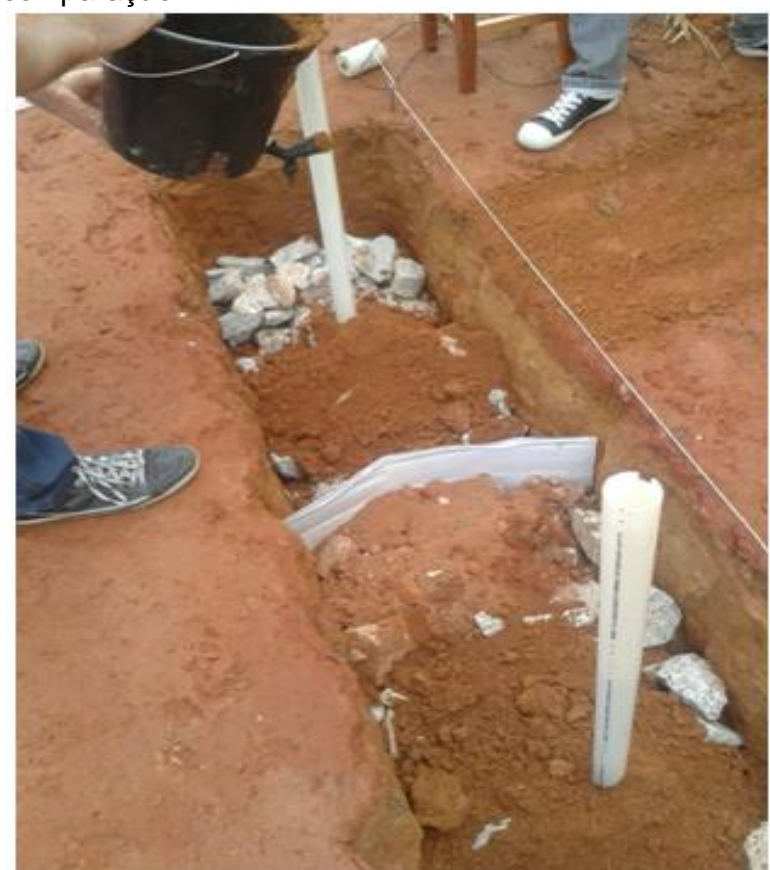

Figura 3 - Preparação do local do experimento

A vala foi preenchida de paralelepípedo (granito) e coberta pelo material do solo, composto predominantemente de argila e os tubos posicionados, conforme mostra a figura (3). Em seguida, delimitado sua área real e posicionando quatro linhas (seções) dispostas paralelamente, com espaçamento de 0,25 m entre os eletrodos, sendo assim possível, mapear quatro níveis de profundidade com até $0,5 \mathrm{~m}$ abaixo do solo. Os tubos foram preparados, figura (4) e fixados no local.
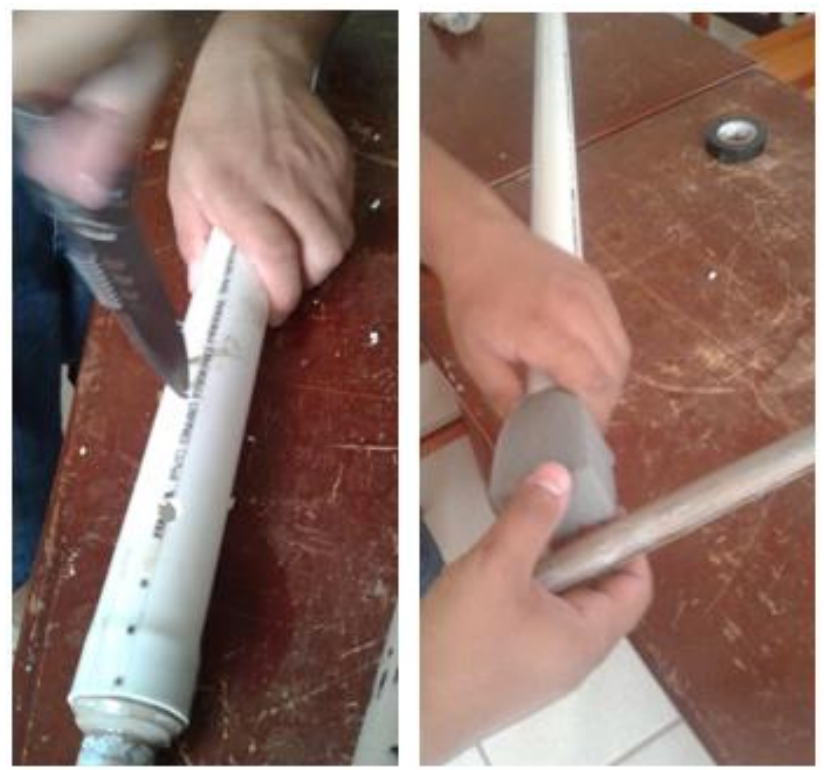

Figura 4 - Preparação dos tubos para realização do experimento. 
Utilizando uma fonte de corrente alternada de $20 \mathrm{~V}$ e um multímetro calibrado em $200 \mu \mathrm{A}$ foi coletado, por meio da técnica do CE (arranjo dipolo-dipolo), uma malha de 94 medidas contendo resultados em ddp (diferença de potencial), para cada linha investigada, dispostas nos quatro níveis.

Após a coleta e organização dos dados, foi calculado o fator geométrico e a resistividade aparente $\left(\rho_{a}\right)$ em cada ponto investigado para obtenção das pseudo seções geoeletricas.

\section{Resultados}

A figura (5) mostra o esquema de distribuição das linhas de medidas do arranjo utilizado. As linhas 1 e 4 são as externas e as linhas 2 e 3 são as centrais. Os tubos por onde foi inserido a solução salina estão entre as linhas 2 e 3 .

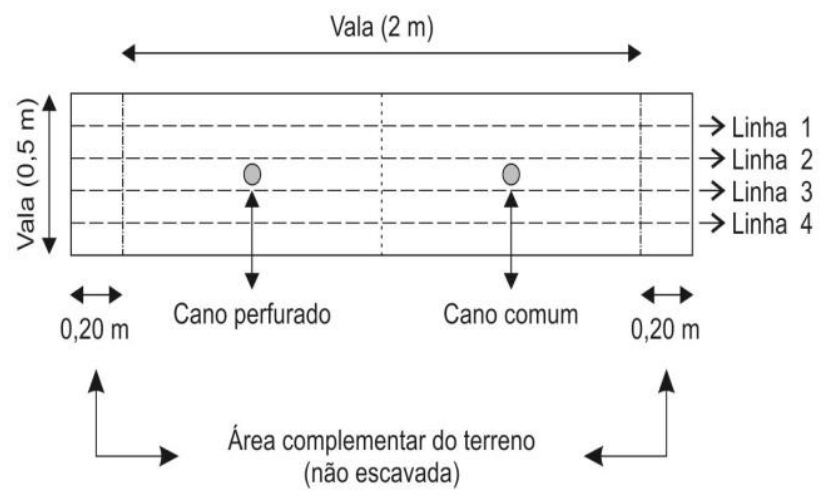

Figura 4 - Esquema das distribuição das linhas para realização da medidas

Após a inserção da solução salina, esperou-se 30 minutos para realização da primeira medição. Uma hora depois foi realizada a segunda medição.

Apresenta-se nas figuras (6a e $6 b$ ) os resultados das pseudo-seções geoelétricas da linha 1. Observa-se que o valor da resistividade aparente diminui sensivelmente entre as duas medições, em função da difusão a solução salina introduzida nos tubos.

As figuras (7a e 7b) mostram os resultados para as pseudo-seções geoelétricas da linha 2 , entre os dois intervalos de tempo e as figuras ( $8 a$ e $8 b)$ as pseudoseções geoelétricas da linha 3 . Pode-se observar uma forte variação da resistividade aparente entre as duas medidas, tanto na linha 2, quanto na linha 3.

As figuras (9a e 9b) expõem o resultado da linha 4. Essa linha apresentou a menor variação entre os valores medidos entre os dois instantes de tempo.

\section{Conclusão}

A experiência descrita nesse trabalho apontou que a resistividade aparente é intensamente afetada pela presença da solução salina. Desta forma, o método geoelétrico da eletrorresistividade, quando aplicado a técnica do Caminhamento Elétrico mostrou-se uma excelente ferramenta para realização de monitoramentos ambientais. E a simulação descrita nesse trabalho pode ser utilizada para treinamento dessa excelente técnica geofísica.

\section{Agradecimentos}

À direção do Instituto de Ciência Engenharia e Tecnologia da Universidade Federal dos Vales do Jequitinhonha e Mucuri, pelo incentivo e orientação no processo de aprendizagem dos métodos geoelétricos. Ao técnico responsável pelo Laboratório de Física, o prof. Fausto Cyrano de Oliveira pela orientação e ajuda no manuseio dos aparelhos elétricos.

\section{Referências}

BRAGA, A. C. O. Geofísica Aplicada - Módulo: Métodos Geoelétricos Aplicados nos Estudos de Captação e Contaminação das Águas Subterrâneas. UNESP - Universidade Estadual Paulista "Julio de Mesquita Filho" - Rio Claro - 2007.

MAGALHÃES JR., A.; MOREIRA, P. F. Eventos de "desequilíbrio morfodinâmico" na evolução da evolução geomorfológica de Minas Gerais - $O$ caso de Teófilo Otoni/Caraí - Região Nordeste do Estado. - UFMG - Universidade Federal de Minas Gerais Geonomos - Vol. 6, no 2, p.23-32; - 1998.

GALLAS, J. D. F. et al. Contaminação por chorume e sua detecção por resistividade. Revista Brasileira de Geofísica, v. 23, n. 1, p. 51-59, jan./mar. 2005.

MOREIRA, C. A.; BRAGA, A. C. de O.. Anomalias de Cargabilidade em Aterro de Resíduos Sólidos Domiciliares. Revista Brasileira de Geofísica, Rio de Janeiro, v. 27, n.1, p. 55-62, jan./mar. 2009.

MOREIRA, C. A.; BRAGA, A. C. de O.; FRIES, M.. Degradação de resíduos e alterações na resistividade elétrica, pH e Eh. Revista Brasileira de Geofísica, Rio de Janeiro, v. 27, n.2, p. 283 - 293, abr./jun. 2009. 


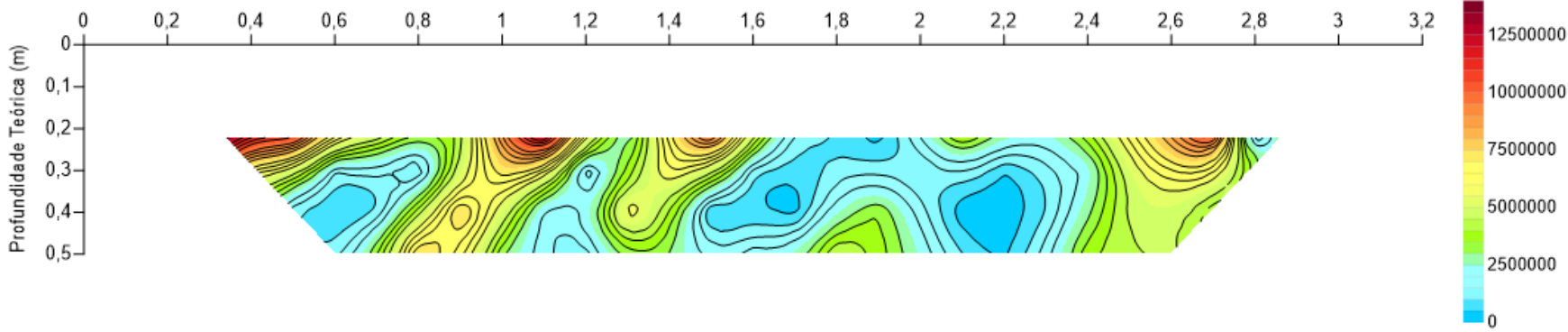

Figura 6a - Pseudo seção geoelétrica da resistividade aparente da linha 1 medição as 08h45min.

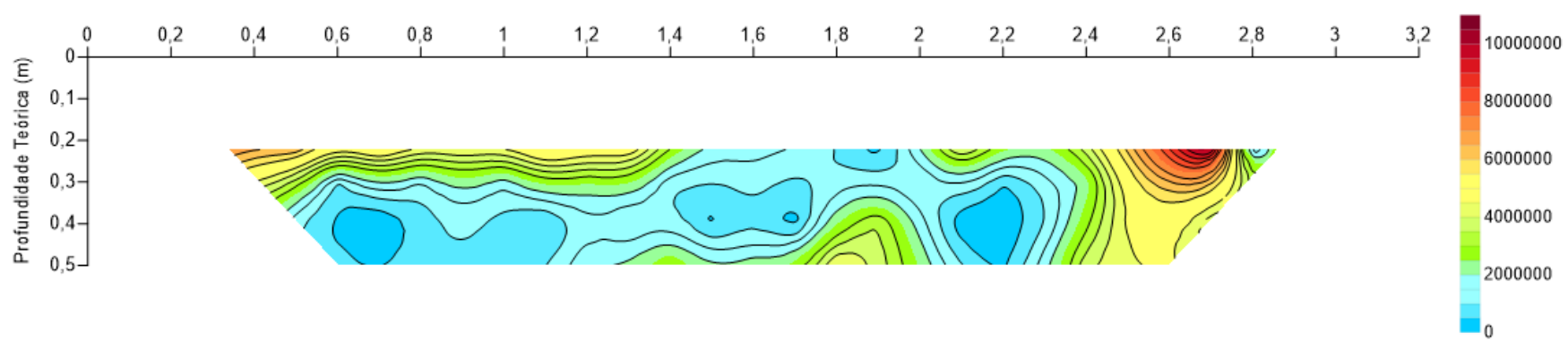

Figura 6b- Pseudo seção geoelétrica da resistividade aparente da linha 1 medição as 09h45min.

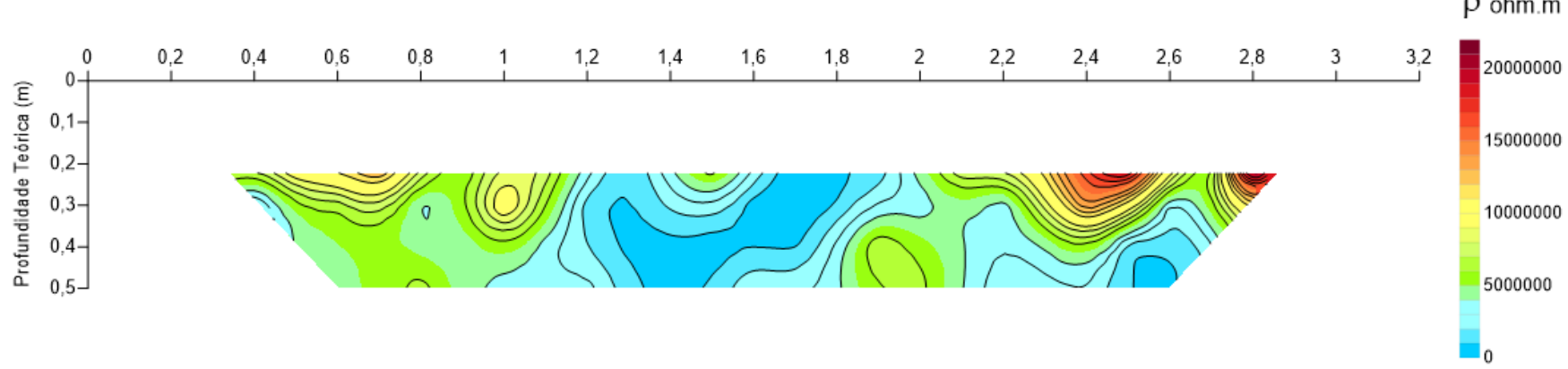

Figura 7a - Pseudo seção geoelétrica da resistividade aparente da linha 2 medição as 08h45min.

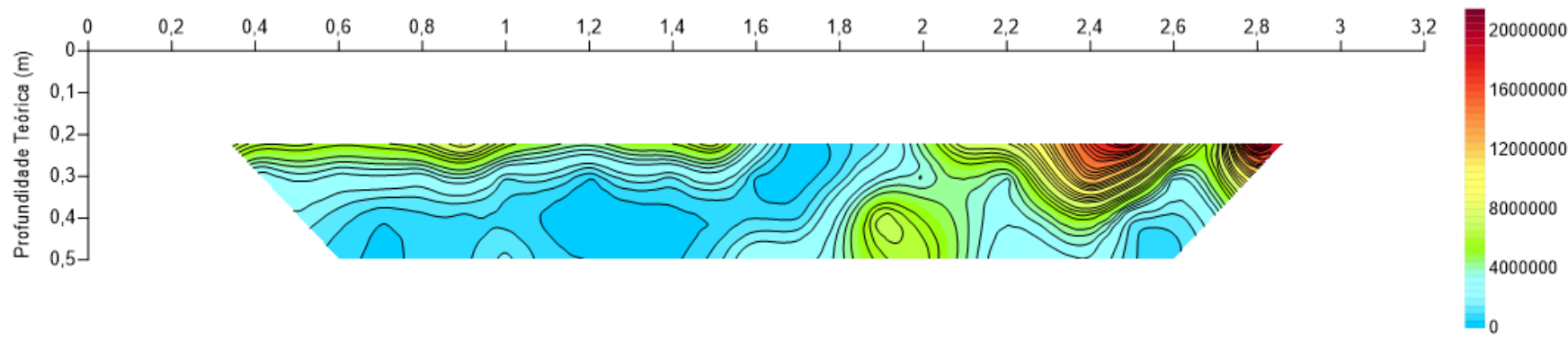

Figura $7 b$ - Pseudo seção geoelétrica da resistividade aparente da linha 2 medição as 09h45min. 


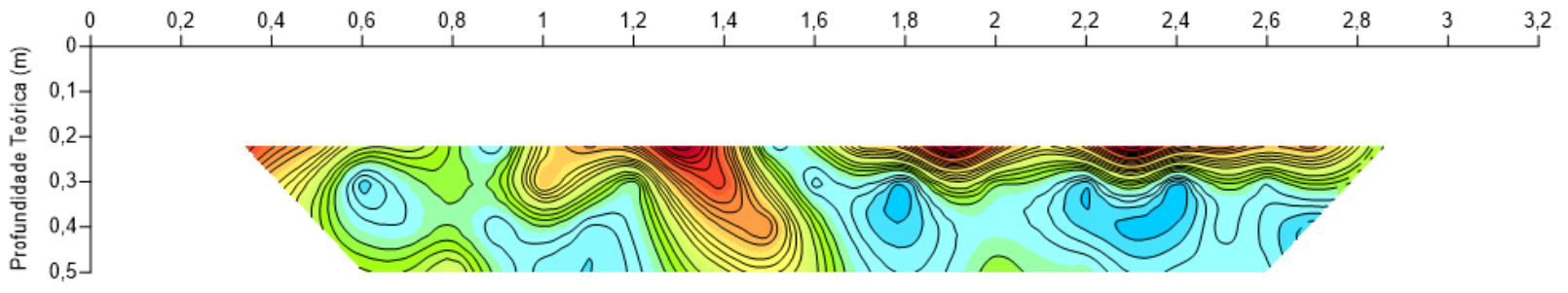

$\rho$ ohm.m

13500000

11000000

8500000

6000000

3500000

$-1000000$

Figura 8a - Pseudo seção geoelétrica da resistividade aparente da linha 3 medição as 08h45min.

$\rho$ ohm.m

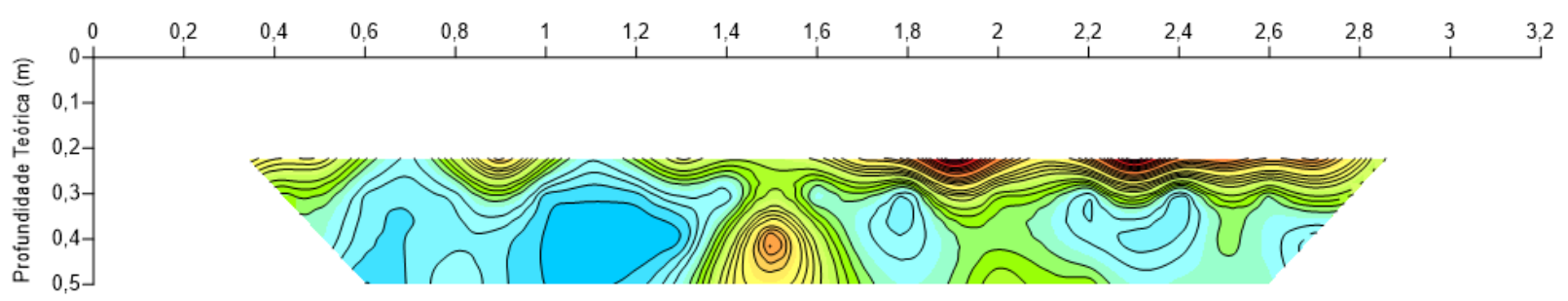

15000000

12000000

9000000

6000000

3000000

Figura 8b - Pseudo seção geoelétrica da resistividade aparente da linha 3 medição as 09h45min.

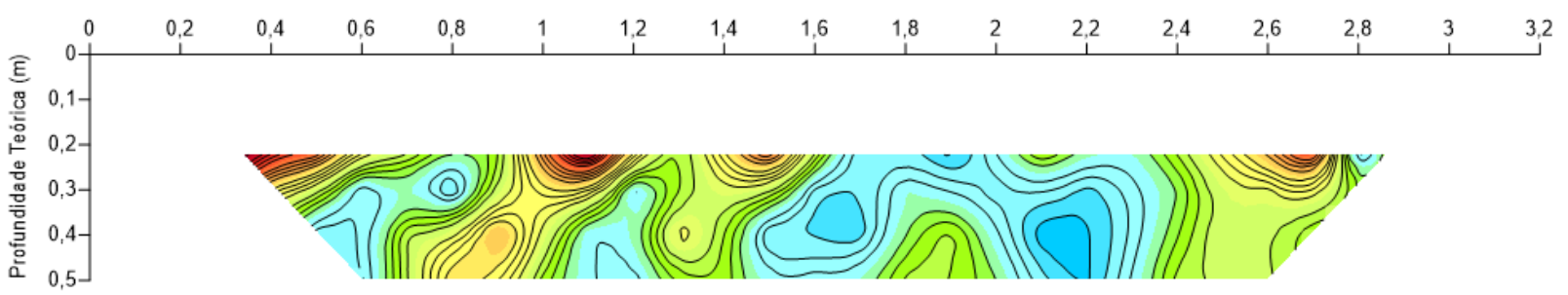

$\rho$ ohm.m

12500000

10000000

7500000

5000000

2500000

Figura 9a - Pseudo seção geoelétrica da resistividade aparente da linha 4 medição as 08h45min.

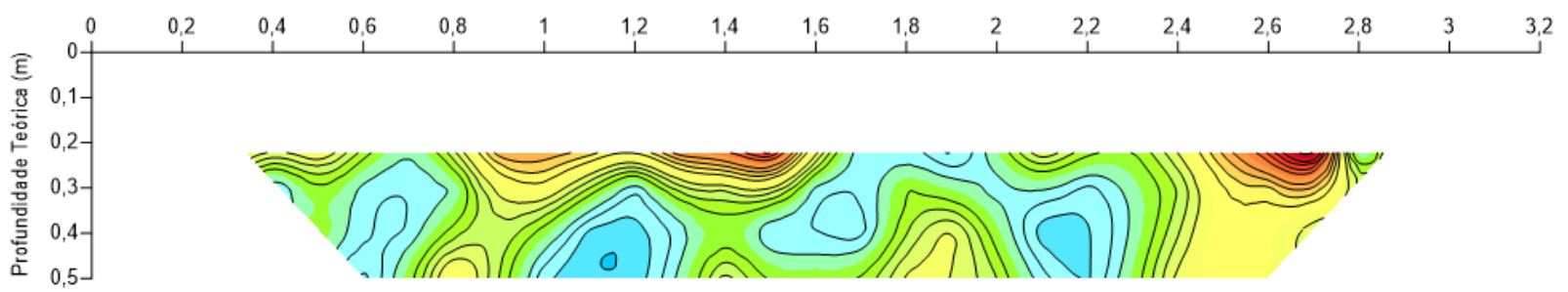

$\rho$ ohm.m

9500000

7000000

4500000

2000000

$-500000$

Figura 9b-Pseudo seção geoelétrica da resistividade aparente da linha 4 medição as 09h45min. 